\title{
Quality of Health Care in Private Nursery Schools at
}

\section{Mansoura City}

\author{
Hoda M. Nafee*, Lamia A. Awad**
}

\begin{abstract}
Childhood is a unique period of rapid growth and development that requires a health system that promotes healthy development of the child. Preschoolers receive care in nursery schools while parents are unavailable due to work or other causes. The care must be affordable, reliable, and accessible. Health services for children exhibit numerous deficiencies in quality of care. The deficiencies are in all major domains of pediatric care, and preventive services. This study was carried out to monitor the quality of health care for preschoolers in private nursery schools. The study was conducted in 8 private nursery schools at Mansoura City by using a systematic random sample. The sample included 607 children, 8 nurses, 48 teachers, and 8 physicians. The data was collected by using two tools. The first tool was used to assess preschoolers' health problems and care provided via medical records. The second tool was used to assess teachers' and nurses' knowledge about preschoolers' health problems and management. An educational program was conducted for nurses and teachers concerning preschoolers' quality health care. The study revealed that there are significant differences in prevalence of preschoolers health problems in relation to educational program. There were significant differences in both nurses' and teachers' knowledge about care of preschoolers after the educational program. All physicians had only a diagnostic role and were not available all of the time. This study recommended that making a significant progress will require not only sustained attention by those concerned about improving child's health and healthcare, but also specific activities to build a broad base of support among the public and key healthcare decision markers.
\end{abstract}

\section{INTRODUCTION}

The early years represent the most critical period of growth and development in child's health(1). In Dakahlia, Egypt (2001), about $12 \%$ of the population were children under 5 years of age ${ }^{(2)}$. Under five years mortality is considered to be one of the key measures of child health ${ }^{(3)}$. United
Nation Development program reported that less than five years mortality rate in Egypt is still higher than its level in other middle income countries(4). Preschool age children are in a relatively healthy period of ife, their most common health problems include acute infectious diseases, *Pediatric Nursing Faculty of Nursing, Mansoura University, Egypt
${ }^{* *}$ Community Health Nursing Faculty of Nursing, Mansoura University, Egypt 
emotional disorders, dental caries, sensory defects, and iron deficiency anemia. They are more active, so they are exposed to accidents which form an important cause of death and disability among them(5).

Nursery school health services include all nursery school activities and procedures designed to deal with the present health status of preschool age children such as appraisal of child health, health counseling, prevention and control of communicable diseases, emergency care, care of handicapped children, in addition to treatment and referral to the health units or hospital. $(6,7)$

In the nursery, school child care is also an arrangement by which adults from outside the family help and those children are sensitive to changes in access to health care services and quality of care $\operatorname{provided}^{(8)}$.

The high quality child care is a community concern and community responsibility, preschool aged children the $\overline{9}$ s will make up to $20 \%$ of the working population in the $21^{\text {st }}$ century(9).

The first event focusing on children's health care since 1997, quality of care was not a prominent theme nationally, and quality held in May 1997(10). In June 1997, article on the future of child health services research, the need to measure and improve quality of children was not a since then key developments in children's health care quality include federal effort ${ }^{(11)}$. A famous statement attributed to Florance Nightingle aptly captures the performance quality measurement relationship "the ultimate goal is to manage quality but you cannot manage it until you have a way to a measure it, and you cannot measure it until you can monitor it(12). Today, quality of care is the degree to which health services for individuals and population increase the likelihood of desired health outcomes and care consistent with current professional knowledge $\mathrm{e}^{(13)}$.

Quality of care can be monitored through 
the first "structure" which refers to health system characteristics that affect the system's ability to meet the health care needs of individual patients or community. Structure indicators describe the type and amount of resources used by a health system or organization to deliver a program and services which are related to the number of staff, clients, beds, supplies, and buildings along with the quantity of money ${ }^{(14,15)}$.

The second "process" which denotes what is actually done in giving and receiving care, i.e., practitioners activities in making a diagnosis, recommending or implementing treatment, or other interaction with patient and the third, "outcome" measures attempt to describe the effects of care on the health status of patients and population and it is a state of health or events that follow care and that may be affected by health care(16).

In Egypt, the health sector reforms often focus on changes in financing or institutional instruction, but neglects a key component which is the staff in the health team $^{(17)}$.

School health nurse facilitates positive student responses to normal development, promotes health and safety, intervenes with actual potential health problems, provides case management services, and activity collaborates with other's to build student and family capacity for adaptation, self-management, self advocacy, and learning ${ }^{(18)}$.

The health sector in Egypt still suffers from a number of problems. These problems include deterioration in the quality of services provided and quality assurance, in addition to nursing problems and shortage of land. Finally, the health insurance in Egypt faces fatal distortions. These problems suggest that health reform in Egypt requires coherent policies not separate ones, besides it will be necessary to establish standard for services provision. 
These standards should include the accreditation requirements for private health units including the facility, staff qualifications, equipment and supplies, as well as record keeping and reporting, recording requirements. Meeting these requirement would be a prerequisite to participating in health sectors ${ }^{(19)}$.

Improving quality and restoring the public confidence in health care needs an individual commitment to make the care one delivers tomorrow better than the care given today, and should learn about improving methods either by reading or attending workshops(20).

Assessing and monitoring quality require attention to both the process and the outcomes of care in order for a process indicator to be valid, it must have been previously demonstrated to produce a better outcome ${ }^{(21)}$.

\section{Aims of the study:}

- Assess the prevalence of preschool aged children health complaining from selected nursery schools.

- Assess the actual health services inside studied nursery schools.

- Assess and enhance the studied nursery school nurses and teachers knowledge about preschool aged children's health complaints and its management via educational program.

\section{MATERIAL AND METHODS}

\section{Setting:}

This study was carried out in 8 private nursery schools equally from East and West Mansoura Educational Departments. It started from September 2005 to May 2006.

\section{Subjects:}

Two classes were selected randomly from each selected private nursery school and those samples were taken as following:

- All preschool aged children from the selected classes $(\mathrm{N}=607)$ were included in this study. Their age, sex, grade, type of complaints, and their 
management.

- All teachers who worked in the selected classes were included in this study $(\mathrm{N}=48)$.

- All nurses and physicians who worked in those private nursery schools were included in this study $(\mathrm{N}=8)$ in each one of them.

Tools of the study: The following tools were used to collect the data in this study:

First. Nursery school records and observation which included 2 parts:

1. Medical record: All preschool aged children who visited the school health clinics from selected classes were assessed for prevalence of health complaints among them and actual care provided by nurses \& physicians, it lasted from (20/9 to 30/12/2005).

2. School health clinics were observed to assess nursery preparation from actual non-human resources inside studied nursery schools and Socio-demographic characteristics of physicians from nursery school record such as age, sex, years of experience, qualification, and observation of actual physician role, attendance, and physician pupils ratio.

Second. Knowledge questionnaire format: after assessment of actual preschool aged children health complaints and school health clinics preparation, the researchers assessed the following:

1- Teachers knowledge about preschool aged children health complaints such as definition, causes, signs and symptoms, management, and complication plus their socio-demographic characteristics such as age, years of experience, qualification, training on first aids, and teacher/pupils ratio $(n=48)$.

2- School nurses knowledge about actual preschool aged children health complaints as mentioned above for teachers plus socio-demographic characteristics of nurse as age, years of experience, qualification, training courses, actual role, and school nurse/pupils ratio $(n=8)$. 
N.B. After assessment of health complaints among preschool aged children and knowledge of teachers and nurses about it, an educational program was performed for nurses and teachers and implemented in the Mid-year holiday. All selected nursery schools gathered in one setting from selected schools in two days. The days start from 9 am to $2 \mathrm{pm}$ in two sessions for two days. Teaching methods were lectures, power point, presentation for clinical pictures about some preschool aged child health complaints and handout for participants about actual health problems of preschool aged children and its management. At the end of the program, the researchers reassess child health complaints and teachers and nurses knowledge about management of it (post test) from 21/1 to 25/4 (pre- and post-test)

\section{Methods:}

Official permission was obtained from Undersecretary of Ministry of Health and
Education in Dakahlia Governorate to carry out this study on 8 private nursery schools (4) from East of Mansoura, El-Hoda Walnour, Faker El-Deen Khalied, El-Tafl Elsaeed, and El-Radwan Eleslamia and (4) from West, Elshaima, El-safa Walmarwa, Omelmoomenen, and EIRahma nursery schools.

\section{Pilot study:}

A pilot study was carried out on 5 teachers from the selected nursery schools and excluded from the study to ascertain the relevance of questions and to detect any problem or defect on the clarity and sequence of the sheet, the questionnaire sheet was reconstructed and become ready to use.

\section{Scoring system:}

\section{Scoring system was used for:}

1) Knowledge assessment: each item took one score and all of those items had total score which was judged as the following, poor $<50 \%$, satisfactory score $50 \%$ to $60 \%$ and good 
score $>60 \%$ according to (Hassaneen 2000). (22)

Actual non-human resources in studied nursery school health clinic in comparing with ideal non-human resources as in total inventory for equipment and supplies in a school health clinic by Anderson (1998)(23) and emergency medication according to the school health insurance book in Egypt (1993).(24) The score of non-human resources in nursery school health clinics were divided into (physical setup $=16$, equipment $=28$, supply for first aid $=12$, and emergency medications = 30). The total non-human resources score was (86) and it was categorized as the following:

- Score $<50 \%$ was considered poor standard.

- Score 50 - <60\% considered fair standard.

- Score 60 - $<75 \%$ considered good standard.

- Score $75 \%$ and above considered very good standard.

\section{Statistical analysis:}

- The first part in statistical analysis was descriptive in the form of frequency and proportions.

- The second part was analysed by using chi-square for comparing statistical differences between two groups or more $\mathrm{P}$ was significant if $<0.05$, confidence interval (95\%).

The statistical analysis of data was done by using SPSS program Statistical Packing of Social Sciences version 10 and Epi-info program on Windows 98.

\section{RESULTS}

Table (1) shows that all preschool aged children (100\%) age range between 4-6 years and also (56.3\%) of them were females, more than half of their fathers and mothers attained higher level of education $(52.7 \%$ and $54.1 \%)$, respectively, concerning the preschool aged child family income of less than half of them (46\%) had slight enough income.

Table (2) indicates that more than one- 
third of preschool aged children (33.3\%) had health complaints (pre-educational program), while the rest had not $(66.7 \%)$ and (post-educational program) was $(26.4 \%)$ while the rest $(73.6 \%)$.

Table (3) shows respiratory complaints among preschool aged children participated (15.7\%) in forms of common cold was the major health complaints among preschool aged children followed by gastrointestinal complaints $(7.1 \%)$ in the forms of abdominal pain, followed by skin complaint (3.8\%) in the forms of skin allergy, (2.5\%), skin infection (1.3\%), then general health complaints, which participate $(3.1 \%)$ in the forms of toothache, eye infection (1.5\%) in addition to injuries (3\%). Statistically no significant differences were found in preschool aged children health complaints throughout educational program.

Table (4) reveals that nearly two-thirds of studied nurses $(62.5 \%)$ their age ranges from 25 - 40 years and the rest (37.5\%) are above (40) years. As regards nurses qualification, all of them had secondary school diploma in nursing, and the majority of them $(87.5 \%)$ their years of experience was less than 15 years and did not receive any training course. All studied nurses had administrative, referral, and curative roles and above half of them (62.5\%) had $1 /<400$ nurses/pupils ratio and the rest (37.5\%) $\operatorname{had} 1 />4000$.

Table (5) indicates that nearly twothirds $(60 \%)$ of nurses had poor level of knowledge about preschool aged children health complaints and its management in pre-educational program and improved to be good level after the implemented program and statistically there were significant differences observed in the nurses level of their knowledge about preschool aged children health complaints through stages of educational program $\mathrm{P}<0.001$.

Table (6) illustrates that nearly twothirds $(62 \%)$ of nursery school physicians 
had their age less than 50 years, ratio, above half of them had 2/45, while concerning years of experience about half one fourth of them had $2 / 70$, and the rest of them $(50 \%)$ had $15-20$ years of (12.5\%) had 2/35.

experience and had diploma in pediatrics,

Table (8) as regards pre-educational while all of them had only a diagnostic role program the level of teachers knowledge and are not available all time at school health clinic.

As regards physician/pupils ratio, about two-thirds $(62.5 \%)$ of them had $1 /<400$ and the rest $(37.5 \%)$ had $1 />4000$.

Table (7) reveals that about half of nursery school teachers age was 25-30 years and less than half of them (45.8\%) had kindergarten specialty while the rest (54.2\%) had no specialty in kindergarten.

As regards their years of experience $(45.8 \%)$ of them had less than one year, while nearly one-fifth of them (20.8\%) had four or more years of experience. Concerning the teachers who attended to first aid training course in pediatrics, this table revealed only (4.2\%) of them attended it, As regards the teacher/pupils about health complaints of preschool aged children, in qualified teacher, it fells between poor and satisfactory while in unqualified teachers it fells in poor level but post-educational program both of them fells in between good and satisfactory level. The differences between two stages are statistically significant $P<0.001$.

Table (9) shows the distribution of nonhuman resources in studied nursery schools by school nurse room according to score of economy.

The table revealed that only three nursery schools (El-Hoda Walnour, ElRadwan El-Eslamia, and Faker El-Deen Khaled) their score for non-human resources were fair $(50 \%$ to $>60 \%$ ) while the rest of them had poor. 
Table (1): Distribution of studied preschool aged children according to their age, sex, and socio-demographic characteristics.

\begin{tabular}{||l|c|c|}
\hline \multicolumn{1}{|c|}{ Items } & No.= 607 & $\%$ \\
\hline \hline \begin{tabular}{|l|l||} 
Age, years: \\
4-6
\end{tabular} & 607 & 100.0 \\
\hline Sex: & 265 & 43.7 \\
Males & 342 & 56.3 \\
Females & & \\
\hline Father education: & 114 & 18.8 \\
Post-graduates & 320 & 52.7 \\
High-education & 69 & 11.3 \\
Above middle education & 74 & 12.2 \\
Middle education & 18 & 3.0 \\
Below middle education & 12 & 2.0 \\
Primary education & & \\
\hline Mother education: & 128 & 21.0 \\
Post-graduates & 328 & 54.1 \\
High-education & 74 & 12.2 \\
Above middle education & 77 & 12.7 \\
Middle education & & \\
\hline Family income: & 159 & 26.2 \\
Enough & 279 & 46 \\
Slightly enough & 169 & 27.9 \\
Not enough & & \\
\hline \hline
\end{tabular}

Table (2): Distribution of health complaints among preschool aged children in private nursery schools in relation to educational program.

\begin{tabular}{|c|c|c|c|c|c|}
\hline \multirow{2}{*}{$\begin{array}{l}\text { Educational program } \\
\text { Preschoolers } \\
\text { health complaints }\end{array}$} & \multicolumn{2}{|c|}{$\begin{array}{l}\text { Pre-educational } \\
\text { program }\end{array}$} & \multicolumn{2}{|c|}{$\begin{array}{l}\text { post-educational } \\
\text { program }\end{array}$} & \multirow{2}{*}{$\begin{array}{l}\text { Chi- } \\
\text { square } \\
\text { test }\end{array}$} \\
\hline & No. $=607$ & $\%$ & No. $=607$ & $\%$ & \\
\hline $\begin{array}{l}\text { Preschooler with } \\
\text { health complaints }\end{array}$ & 202 & 33.3 & 160 & 26.4 & \multirow{3}{*}{$\begin{array}{l}\chi^{2}=6.62 \\
P=0.01 \text { * }\end{array}$} \\
\hline $\begin{array}{l}\text { Preschoolers without } \\
\text { health complaints }\end{array}$ & 405 & 66.7 & 447 & 73.6 & \\
\hline Total & 607 & 100 & 607 & 100 & \\
\hline
\end{tabular}

N.B: ${ }^{*}=$ highly significant differences 
Table (3): Prevalence of health complaints among preschool aged children according to their body systems in relation to educational program.

\begin{tabular}{|c|c|c|c|c|c|}
\hline \multirow{2}{*}{$\begin{array}{l}\text { Preschoolers } \\
\text { health complaints }\end{array}$} & \multicolumn{2}{|c|}{$\begin{array}{l}\text { Pre-educational } \\
\text { program }\end{array}$} & \multicolumn{2}{|c|}{$\begin{array}{c}\text { post-educational } \\
\text { program }\end{array}$} & \multirow{2}{*}{$\begin{array}{c}\text { Chi-square } \\
\text { test }\end{array}$} \\
\hline & No. $=607$ & $\%$ & No. $=607$ & $\%$ & \\
\hline Respiratory complaints & & & & & $\chi^{2}=8.3$ \\
\hline - Common cold & 95 & 15.7 & 69 & 11.4 & \\
\hline - Bronchial asthma & 4 & 0.7 & 2 & 0.3 & $P=0.04^{*}$ \\
\hline Subtotal & $\overline{99}$ & 16.3 & $\overline{71}$ & 11.7 & \\
\hline $\begin{array}{c}\text { Gastrointestinal complaints } \\
\bullet \text { Abdominal pain }\end{array}$ & 43 & 7.1 & 32 & 5.3 & $\begin{array}{c}5.03 \\
P=0.08\end{array}$ \\
\hline Subtotal & 43 & $\overline{7.1}$ & 32 & 5.3 & \\
\hline $\begin{array}{l}\text { Skin complaints } \\
\bullet \text { Skin allergy } \\
\bullet \text { Skin infection }\end{array}$ & $\begin{array}{c}15 \\
8\end{array}$ & $\begin{array}{l}2.5 \\
1.3\end{array}$ & $\begin{array}{c}9 \\
11\end{array}$ & $\begin{array}{l}1.5 \\
1.8\end{array}$ & $\begin{array}{l}\chi^{2}=5.5 \\
P=0.13\end{array}$ \\
\hline Subtotal & 23 & 3.8 & 20 & 3.3 & \\
\hline Emergency injuries & 18 & 3.0 & 19 & 3.1 & $\begin{array}{l}\chi^{2}=1.21 \\
P=0.5\end{array}$ \\
\hline Subtotal & 18 & 3 & 19 & 3.1 & \\
\hline $\begin{array}{c}\text { General complaints } \\
\text { • Tooth ache } \\
\text { • Eye infection }\end{array}$ & $\begin{array}{c}10 \\
9\end{array}$ & $\begin{array}{l}1.6 \\
1.5\end{array}$ & $\begin{array}{c}10 \\
8\end{array}$ & $\begin{array}{l}1.6 \\
1.2\end{array}$ & $\begin{aligned} \chi^{2} & =0.08 \\
P & =9.5\end{aligned}$ \\
\hline Subtotal & 19 & 3.1 & 18 & 2.8 & $\chi^{2}=1.64$ \\
\hline Total & 202 & 33.3 & 160 & 26.4 & $P=0.802$ \\
\hline
\end{tabular}

${ }^{*}$ significant

Table (4): Distribution of the nursery school nurses by their personal and work characteristics.

\begin{tabular}{|c|c|c|}
\hline Items & No. $=8$ & $\%$ \\
\hline $\begin{array}{c}\text { Age, years } \\
25- \\
40+ \\
\end{array}$ & $\begin{array}{l}5 \\
3 \\
\end{array}$ & $\begin{array}{l}62.5 \\
37.5 \\
\end{array}$ \\
\hline $\begin{array}{l}\text { Qualification } \\
\quad \text { Secondary nursing school diploma }\end{array}$ & 8 & 100.0 \\
\hline $\begin{array}{c}\text { erience, years } \\
5- \\
15-20\end{array}$ & $\begin{array}{l}7 \\
1\end{array}$ & $\begin{array}{l}87.5 \\
12.5\end{array}$ \\
\hline $\begin{array}{l}\text { Training courses received } \\
\text { Yes } \\
\text { No } \\
\text { Topic (first aid) }\end{array}$ & $\begin{array}{l}1 \\
7 \\
1\end{array}$ & $\begin{array}{l}12.5 \\
87.5 \\
12.5 \\
\end{array}$ \\
\hline $\begin{array}{l}\text { Roles of school nurses } \\
\text { Administrative, referral, and curative roles }\end{array}$ & 8 & 1000 \\
\hline $\begin{array}{c}\text { School nurse/pupils ratio } \\
1 /<400 \\
1 />4000\end{array}$ & $\begin{array}{l}5 \\
3 \\
\end{array}$ & $\begin{array}{l}62.5 \\
37.5 \\
\end{array}$ \\
\hline
\end{tabular}


Table (5): Percentage distribution of studied nurses level of knowledge related to health complaints among preschool aged children via educational program (pre and post).

\begin{tabular}{|c|c|c|c|c|c|}
\hline \multirow{2}{*}{\begin{tabular}{|l|} 
\\
$\begin{array}{l}\text { Preschoolers } \\
\text { health complaints }\end{array}$ \\
\end{tabular}} & \multicolumn{2}{|c|}{$\begin{array}{c}\text { Pre-educational } \\
\text { program }\end{array}$} & \multicolumn{2}{|c|}{$\begin{array}{c}\text { post-educational } \\
\text { program }\end{array}$} & \multirow{2}{*}{$\begin{array}{c}\text { Chi-square } \\
\text { test }\end{array}$} \\
\hline & No.=8 & $\%$ & No. $=8$ & $\%$ & \\
\hline \multicolumn{6}{|l|}{ Respiratory complaints } \\
\hline - Good & - & 0.0 & 5 & 62.5 & $\chi^{2}=10$ \\
\hline - Satisfactory & 3 & 37.5 & 3 & 37.5 & $P=0.006^{* *}$ \\
\hline - Poor & 5 & 62.5 & 0 & 0.0 & \\
\hline $\begin{array}{c}\text { Gastrointestinal complaints } \\
- \text { Good } \\
\text { - Satisfactory } \\
\text { - Poor }\end{array}$ & $\begin{array}{l}1 \\
2 \\
5\end{array}$ & $\begin{array}{l}12.5 \\
25.0 \\
62.5\end{array}$ & $\begin{array}{l}5 \\
3 \\
0\end{array}$ & $\begin{array}{c}62.5 \\
37.5 \\
0.0\end{array}$ & $\begin{array}{c}\chi^{2}=9.83 \\
\mathrm{P}=0.007^{\star *}\end{array}$ \\
\hline $\begin{array}{c}\text { Emergency injuries } \\
\bullet \text { Good } \\
\bullet \text { Satisfactory } \\
\bullet \text { Poor } \\
\end{array}$ & $\begin{array}{l}1 \\
2 \\
5 \\
\end{array}$ & $\begin{array}{l}12.5 \\
25.0 \\
62.5 \\
\end{array}$ & $\begin{array}{l}7 \\
1 \\
0 \\
\end{array}$ & $\begin{array}{c}87.5 \\
12.5 \\
0.0 \\
\end{array}$ & $\begin{array}{l}\chi^{2}=9.83 \\
P=0.007^{\star *}\end{array}$ \\
\hline $\begin{array}{l}\text { Skin complaints } \\
\bullet \text { Good } \\
\bullet \text { Satisfactory } \\
\bullet \text { Poor }\end{array}$ & $\begin{array}{l}0 \\
2 \\
6\end{array}$ & $\begin{array}{c}0.0 \\
25.0 \\
75.0 \\
\end{array}$ & $\begin{array}{l}6 \\
2 \\
0 \\
\end{array}$ & $\begin{array}{c}75.0 \\
25.0 \\
0.0 \\
\end{array}$ & $\begin{array}{c}\chi^{2}=12 \\
P=0.002^{\star *}\end{array}$ \\
\hline $\begin{aligned} \text { General complaints } \\
\\
\bullet \text { Good } \\
\bullet \text { Satisfactory } \\
\text { • Poor }\end{aligned}$ & $\begin{array}{l}0 \\
0 \\
8\end{array}$ & $\begin{array}{c}0.0 \\
0.0 \\
100.0\end{array}$ & $\begin{array}{l}5 \\
2 \\
1\end{array}$ & $\begin{array}{l}62.5 \\
25.0 \\
12.5\end{array}$ & $\begin{array}{l}\chi^{2}=12.44 \\
P=0.001^{\star *}\end{array}$ \\
\hline
\end{tabular}

Table (6): Distribution of the studied private nursery school physicians by their personal and work characteristics.

\begin{tabular}{|l|c|c|}
\hline \multicolumn{1}{|c|}{ Items } & No.= 8 & $\%$ \\
\hline \hline Age, years & 5 & 62.5 \\
$40-$ & 3 & 37.5 \\
$50+$ & & 50.0 \\
\hline Experience, years & 4 & 37.5 \\
$15-$ & 3 & 12.5 \\
$20-$ & 1 & 37.5 \\
$35+$ & & 50.5 \\
Ilification & 3 & 12.5 \\
General practitioner & 4 & 100.0 \\
Diploma in pediatrics & 1 & 100.0 \\
Doctorate in pediatrics & & \\
\hline Actual physician roles & 8 & 100.0 \\
Diagnostic & 8 & 62.5 \\
Not follow up role & & 37.5 \\
\hline Availability all time & 8 & \\
None & & \\
\hline Physician/pupils ratio & 5 & \\
1/<400 & 3 & \\
1/> 400 & & \\
\hline
\end{tabular}


Table (7): Distribution of private nursery schools teachers by their personal and work characteristics.

\begin{tabular}{|c|c|c|}
\hline Items & No. $=48$ & $\%$ \\
\hline $\begin{array}{c}\text { Age / years } \\
20- \\
25- \\
30+\end{array}$ & $\begin{array}{c}20 \\
24 \\
4 \\
\end{array}$ & $\begin{array}{r}41.7 \\
50.0 \\
8.3\end{array}$ \\
\hline $\begin{array}{l}\text { Qualification } \\
\text { Graduated from kindergarten } \\
\text { Higher education } \\
\text { Above middle education } \\
\text { Middle education }\end{array}$ & $\begin{array}{l}22 \\
12 \\
6 \\
8\end{array}$ & $\begin{array}{l}45.8 \\
25.0 \\
12.5 \\
16.7\end{array}$ \\
\hline $\begin{array}{l}\text { Experience, years } \\
>1 \\
1- \\
2- \\
3- \\
4+ \\
\end{array}$ & $\begin{array}{c}22 \\
2 \\
10 \\
4 \\
10\end{array}$ & $\begin{array}{r}45.8 \\
4.1 \\
20.8 \\
8.3 \\
20.8\end{array}$ \\
\hline $\begin{array}{l}\text { Attendance courses about first aids } \\
\text { Yes } \\
\text { No }\end{array}$ & $\begin{array}{c}2 \\
46 \\
\end{array}$ & $\begin{array}{r}4.2 \\
95.8\end{array}$ \\
\hline $\begin{array}{c}\text { Teacher/Pupils ratio } \\
2 \text { / } 70 \\
2 \text { / } 45 \\
2 \text { / } 35\end{array}$ & $\begin{array}{c}12 \\
30 \\
6\end{array}$ & $\begin{array}{l}25.0 \\
62.5 \\
12.5\end{array}$ \\
\hline
\end{tabular}

Table (8): Distribution of teachers' level of knowledge according to their qualification about health complaints of preschool aged children via educational program.

\begin{tabular}{|c|c|c|c|c|c|c|c|c|c|}
\hline \multirow{3}{*}{$\begin{array}{l}\text { Stage and teacher } \\
\text { Qualification } \\
\text { Child complaints }\end{array}$} & \multicolumn{4}{|c|}{ Pre-educational program } & \multicolumn{4}{|c|}{ post-educational program } & \multirow{3}{*}{$\begin{array}{l}\text { Chi- } \\
\text { square } \\
\text { test }\end{array}$} \\
\hline & \multicolumn{2}{|c|}{ Qualified } & \multicolumn{2}{|c|}{ Not qualified } & \multicolumn{2}{|c|}{ Qualified } & \multicolumn{2}{|c|}{ Not qualified } & \\
\hline & No. $=22$ & $\%$ & No. $=26$ & $\%$ & No. $=22$ & $\%$ & No. $=26$ & $\%$ & \\
\hline Respiratory complaints & & & & & & & & & \\
\hline - Good & 0 & 0.0 & 0 & 0.0 & 22 & 100.0 & 26 & 100.0 & $\chi^{2}=70$ \\
\hline - Satisfactory & 2 & 9.1 & 0 & 0.0 & 0 & 0.0 & 0 & 0.0 & $001^{*}$ \\
\hline - Poor & 20 & 90.9 & 26 & 100.0 & 0.0 & 0.0 & 0 & 0.0 & \\
\hline $\begin{array}{c}\text { Gastrointestinal complaints } \\
\text { - Good } \\
\text { - Satisfactory } \\
\text { - Poor }\end{array}$ & $\begin{array}{c}0 \\
4 \\
18 \\
\end{array}$ & $\begin{array}{c}0.0 \\
18.2 \\
81.8 \\
\end{array}$ & $\begin{array}{c}0 \\
0 \\
26 \\
\end{array}$ & $\begin{array}{c}0.0 \\
0.0 \\
100.0 \\
\end{array}$ & $\begin{array}{c}22 \\
0 \\
0\end{array}$ & $\begin{array}{c}100.0 \\
0.0 \\
0.0 \\
\end{array}$ & $\begin{array}{c}22 \\
4 \\
0 \\
\end{array}$ & $\begin{array}{c}84.6 \\
15.4 \\
0.0 \\
\end{array}$ & $\begin{array}{c}\chi^{2}=83.9 \\
P=0.0001^{*}\end{array}$ \\
\hline $\begin{array}{l}\text { Skin complaints } \\
\cdot \text { Good } \\
\cdot \text { Satisfactory } \\
\cdot \text { Poor } \\
\end{array}$ & $\begin{array}{c}0 \\
12 \\
10 \\
\end{array}$ & $\begin{array}{c}0.0 \\
54.6 \\
45.4 \\
\end{array}$ & $\begin{array}{c}0 \\
0 \\
26 \\
\end{array}$ & $\begin{array}{c}0.0 \\
0.0 \\
100.0 \\
\end{array}$ & $\begin{array}{c}20 \\
2 \\
0 \\
\end{array}$ & $\begin{array}{l}90.9 \\
9.1 \\
0.0 \\
\end{array}$ & $\begin{array}{l}16 \\
6 \\
4 \\
\end{array}$ & $\begin{array}{l}61.5 \\
23.1 \\
15.4 \\
\end{array}$ & $\begin{array}{l}\chi^{2}=62.4 \\
\mathrm{P}=0.001^{*}\end{array}$ \\
\hline $\begin{array}{c}\text { Emergency injuries } \\
\cdot \text { Good } \\
\text { - Satisfactory } \\
\text { • Poor } \\
\end{array}$ & $\begin{array}{c}0 \\
10 \\
12 \\
\end{array}$ & $\begin{array}{c}0.0 \\
45.4 \\
54.6 \\
\end{array}$ & $\begin{array}{c}0 \\
0 \\
26 \\
\end{array}$ & $\begin{array}{c}0.0 \\
0.0 \\
100.0 \\
\end{array}$ & $\begin{array}{c}22 \\
0 \\
0 \\
\end{array}$ & $\begin{array}{c}100.0 \\
0.0 \\
0.0 \\
\end{array}$ & $\begin{array}{c}16 \\
8 \\
2 \\
\end{array}$ & $\begin{array}{c}61.5 \\
30.8 \\
7.7 \\
\end{array}$ & $\begin{array}{c}\chi^{2}=70.62 \\
P=0.0001^{*}\end{array}$ \\
\hline $\begin{aligned} & \text { General } \text { complaints } \\
& \text { - Good } \\
& \text { - Satisfactory } \\
& \text { - Poor }\end{aligned}$ & $\begin{array}{c}0 \\
2 \\
20\end{array}$ & $\begin{array}{c}0.0 \\
9.1 \\
90.9\end{array}$ & $\begin{array}{c}0 \\
0 \\
26 \\
\end{array}$ & $\begin{array}{c}0.0 \\
0.0 \\
100.0\end{array}$ & $\begin{array}{c}20 \\
2 \\
0\end{array}$ & $\begin{array}{c}90.9 \\
9.1 \\
0.0 \\
\end{array}$ & $\begin{array}{c}14 \\
10 \\
2 \\
\end{array}$ & $\begin{array}{c}53.8 \\
38.5 \\
7.7\end{array}$ & $\begin{array}{l}\chi^{2}=71.28 \\
P=0.0001^{*}\end{array}$ \\
\hline
\end{tabular}




\begin{tabular}{|c|c|c|c|c|c|c|c|c|c|c|}
\hline & eeu!us-Iヨ & å & 융 đู & & 空 & 冓 & 㟋 & $\frac{0}{\dot{y}}$ & ت্ & $\stackrel{\infty}{\dot{y}}$ \\
\hline & & 울 & $-\operatorname{son}$ & & - & $\nabla$ & on & مs & $\infty$ & ల \\
\hline & емиешіеM ejes-l & $\therefore$ & 융유 & & 잉 & n & 尔 & $\frac{0}{8}$ & $\widetilde{0}$ & एా \\
\hline 응 & & 운 & $-\forall n$ & & 0 & in & in & $n$ & $\infty$ & $m$ \\
\hline 心్ర & קו-4 & $\therefore$ & 융무욤 & & 응 & 隹 & 尔 & $\frac{0}{8}$ & స్ల & क্ల \\
\hline 음 & ב & 울 & 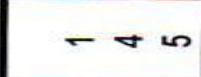 & & 0 & on & on & o & $\infty$ & ల్ల \\
\hline 응 & чәәищ & $\therefore$ & 융유뮴 & & బั) & 㐫 & 每 & $\frac{0}{8}$ & हृ & 禜 \\
\hline हूँ & 응 & 울 & $-\nabla \infty$ & & - & ナ & in & n & $\infty$ & m \\
\hline 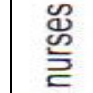 & 焉 & se & 융 范 & & 오. & 象 & @. & 유 & 웅 & 出 \\
\hline$\frac{0}{8}$ & $\sum$ & 운 & $-N \omega$ & & $\sim$ & 60 & $n$ & 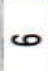 & $\stackrel{2}{=}$ & \& \\
\hline$\sum_{\substack{\infty \\
\infty}}^{\infty}$ & & a & 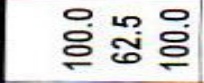 & & సें & के & 尔 & $\frac{0}{8}$ & ت্ণ & $\frac{\infty}{8}$ \\
\hline 름 & IE & i் & $-\ln \pi$ & & -7 & $\nabla$ & in & in & $\infty$ & జ్ల \\
\hline $\begin{array}{l}\text { 을 } \\
\text { 에 } \\
\text { 는 }\end{array}$ & 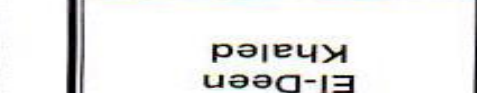 & a & 융 ฮึ 융 & & 유. & $\begin{array}{l}2 \\
0 \\
0\end{array}$ & 管 & $\frac{0}{8}$ & 윰 & 芦 \\
\hline$\cong$ & AyXey & i & $-\operatorname{son}$ & & $\sim$ & $\infty$ & 0 & in & 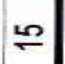 & $\approx$ \\
\hline 을 & AnoujeM e & 20 & 응 م & & 유: & م & 每 & 웅 & 웅 & డ్ర \\
\hline 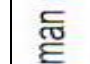 & & 울 & $-r$ & & $\sim$ & c & 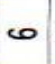 & $\omega$ & $\cong$ & 유 \\
\hline 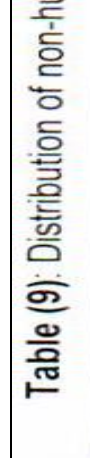 & 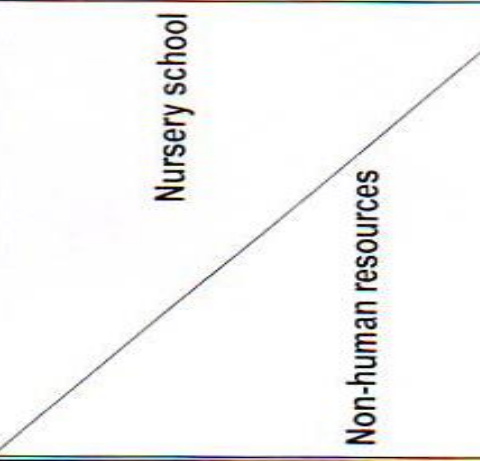 & & 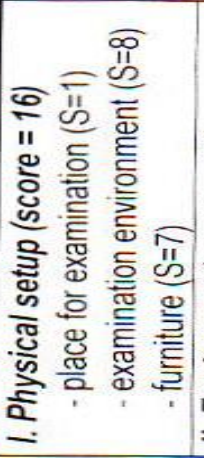 & 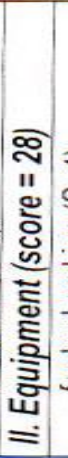 & 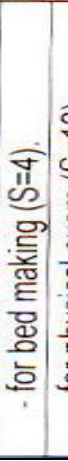 & 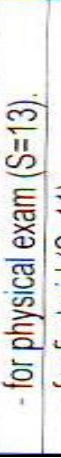 & 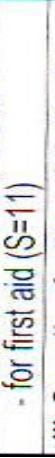 & 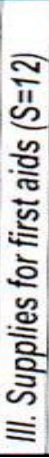 & 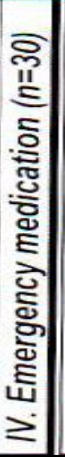 & 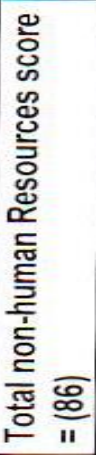 \\
\hline
\end{tabular}




\section{DISCUSSION}

The number of children at preschool aged is increasing in most countries throughout the world(25). World Health Organization pointed out that the marked increase in number of children at preschool aged is greatly associated with a more proportion increase in the demanding and using of a adequate health services. ${ }^{(26)}$

Children under 5 years old were more likely to have visited a health professional or been hospitalized, they are sensitive to change in access to health care services and quality of care provided(27,28), they are more exposed to variable health problems such as communicable diseases, deficiency diseases, psychological disturbances, aliments of special senses, and accident.

Catalono and Ory (1997) $)^{(29)}$ emphasized that the assessment of health care quality does not reside either for structure, process, or outcome, but the link between the three.
As regards preschool child outcome in the present study pre-educational program revealed that about $(33.3 \%)$ of them had health complaints and the main complaint had upper respiratory tract infection (16.3\%) and this result in agreement with Waller (2001)(30) who found that children attending day-care centers are at risk of infection in their upper respiratory systems and also Lauber $(2005)^{(31)}$ found that $(11 \%)$ of preschool aged children in the day care center suffered from upper respiratory tract infection in the form of sore throat, pharyngitis, tonsillitis, sinusitis, and acute otitis media.

In addition, Pelletier et al., (2004)(32) found that $(33 \%)$ of diseased children under 5 years were related to environmental exposure, this result agrees with the present study where in the majority of children, their parents were busy due to their work, increase number of children in their classes, decrease number of 
teachers, nurses and physicians in relation to children numbers.

The second preschool aged children health complaints in the present study was abdominal pain as it constituted about (7.1\%) and this result agrees with Rit and Ravinderk (2004)(33) who found that recurrent abdominal pain among preschool aged was about (10-15\%).

Peronic and Pia Centiri (2003)(34) found that common cold (16.8\%) and skin dermatitis $(12.3 \%)$ represented the major health complaints among preschool aged children which predispose to asthma $(20.3 \%)$. This result disagrees with the finding of the present study which showed that skin dermatitis (2.5\%) and asthma $(0.7 \%)$ represented the minor health complaints among preschool aged children.

\section{American Academy of Pediatrics} $(2004)^{(35)}$ mentioned that children and youth can be injured or become ill during the school day, and may require nonurgent, urgent, or emergency health care at school, also Kersting and Mielck $(2002)^{(36)}$ found that more than forth $(26 \%)$ of kindergartens had accidents.

Improvment of quality of services through appropriate allocation of the resources, compliance with standards of case management, counseling, preventive activities, and referral (MOHP, 1199)(37).

Individuals should be responsible for fairly judgment of the quality of health services communicating their evaluation to the providers in a manner conductive to the maintenance of quality (37).

Walker $(2001)^{(38)}$ found that raining and educating child care providers on preventing injuries and illnesses improve the safety and health of the child and this study disagrees with the present study in the parts related to prevention of injuries where pre-educational program child injures was (3\%) while increased by $(3.1 \%)$ after the implementation of educational program for children care giver while 
agree in the other preschool children illness which decrease after the implementation of educational program for children care giver.

World Health Organization (2006) ${ }^{(39)}$ found that human recourses improvement is a key issue in reforming health sector, as they are candidates who apply this reform. Thus, improvement should be reflected in enhancing work conditions, education, and continuous training.

Bethell et al., (2003)(40) confirm that there is a need for ongoing effort to bridge the gab between guidelines and practices in the area of early childhood care, preventive, developmental services, and barriers to routinely incorporating developmental services into practices may include a lack of health care provider knowledge, time, economic, incentives, or training, the above mentioned studies agree with the present study where care giver for preschool aged children as nurses, and teachers, their knowledge about preschool aged health complaints and its management need enhancement.

Bassil, et al., (2000)(41) mentioned that regular monitoring of health system performance is warranted along with emphasis on health education program for caretakers and care givers of children with chronic diseases about improving quality of care and outcome.

Gunter and Matteson (2001) mentioned that continuing education programs are designed to help staff to keep up to-date with new concepts and acquire greater depth of knowledge. Also programs are responsible for changing attitude and values, implementing concepts of change, both within the individual own practice and throughout the health care delivery system.

The study agrees with the present study where level of knowledge of care givers for preschool aged children about their health complaints and its management improved 
from poor to good and satisfactory level in relation to pre- and post-health educational program.

Harriagan (2002)(43) emphasized that school health nurse promotes health by providing program to staff, families, health fairs for staff, parents of the child, and consultation with other school staff. This study disagrees with the present study where school health nurses had not a health educator role. In the United States, the Department of Human Health Services $(2000)^{(44)}$ recommended that at least one nurse for 750 students with variation depending on the country and student population, this result disagrees with the present study where the nurses/pupils ratio ranged between $<400$ to $>400$.

In Soviet Medicine (1999) the main task was formation of a populations healthy life style, which can be accomplished with the help of school education team. Another study done by Telljohann (1999)(45) who found that most teachers were self-taught with regard to health education as there is no formal training and relied primarily on traditional teacher centered instruction methods. In services training there is available for teachers. Also in Bahrain AlNasier and Sherman (2004)(46) found that teacher's health knowledge was deficient and this may ultimately affect their ability either to deliver health education or to mange acute health problems in their schools. The two previous studies agree with the present study where $95.8 \%$ of nursery school teachers did not attend training course about child first aids and all of their knowledge about care of preschool aged child health problems had ranged between poor and satisfactory level.

Mark et al., (2002)(47) emphasized that current effort to improve health care will not succeed unless we undertake a major systemic effort to overhaul how we deliver health care services, educate, and improve quality .

Rajmil et al., (2005) ${ }^{(48)}$ found that study 
of the quality of health services and monitoring of the evaluation of these unequalities are important matters of concern and also in Egypt Saker and Heiba $(2006)^{(17)}$ found that health services improvement must not be restricted only to increase the coverage of health care but also include improving the quality of health services provided in addition to health system which suffers from shortage in coverage of emergency services as well as deterioration of quality of health services. The above mentioned study in agreement with present study where health services provided for preschool aged children were poor and deteriorated.

\section{CONCLUSION}

Health and well being of our children should be a national priority. In the data of this study revealed that:

1) Quality of health care for children was far less than optimal

2) Human resources had no appropriate qualification for meeting the child health care needs.

3) Increase number of children in relation to health care team number, no training program for improving knowledge of caregivers.

4) Un-availability of physicians in all time inside nursery schools plus.

5) Poor non-human resources in the majority of nursery. schools

6) No health care plan and follow-up care for children with health complaints and also caregivers had no role in health teaching or health promotion.

7) There was a slight improvement of children health status due to complicated process of quality health care inside nursery schools.

\section{RECOMMENDATIONs}

In view of the previous conclusions, the following recommendations are suggested:

- The team work in the nursery school should be qualified to meet child needs.

- Develop of quality measures and 
implementing of quality improvement

strategies for children's health care.

- Build a broad base of support among

the public and key health care decision

makers to improve the quality of health

care.

- Preventive services on the basis of national standards, practice teams, attended a quality improvement workshop, learning collaborations are potentially effective methods for improving the quality of care that delivered by the health care providers.

\section{REFERENCES}

1. Richard EB, Robert MK, Hal BJ. Nelson text book of pediatrics, $16^{\text {th }}$ ed. Philadelphia: W.B. Sandurs Comp; 2004. 213-216.

2. El - Gilany AH, Hammed S. Epidemiology of diarrheal disease among children under 5 years. Dakahlia Governorate, Egypt: 2001. $115-120$.

3. World Human Development Report. New York; 2005. 13 - 18.

4. UNICEF. The state of the world's children "children under threat". 2005. $116-120$.

5. Young PC, Glade GB, Stodder GJ, Norlin C. Evaluation of a learning collaboration to improve the delivery services by pediatric practices. Pediatrics. 2006;117 (5):1469 - 76.
6. Satcher D, Bradford M. Healthy schools, health kids. Am School Braad Journal. 2003; 190 (3): $22-5$.

7. Bredkamps. Accreditation criteria proceducrs. National Academy of Early Childhood Programs. Washington, DC: National Association for the Education of Young children; (2000). 215 - 220.

8. Luis RL, Caime B, Barbara S. The quality of care and influence of double health care coverage in Catalonia Spain (2000): Arch Dis Child. 2000;83: 211-4.

9. Brederamp S. Developmentally appropriate practices in early childhood services: children from birth throughout age of 8 years. Washington DC: National Association of the Education of Young Children; 1999. 118 - 132.

10. Forrest, C, Simpson L, Cloncy C. Child health services research: challenge and opportunities. JAMA. 1997; 227: 1787-93.

11. Center for Medicare and Medicaid Services. Framework for annual report of state children's health, insurance programs under title XXI of the social security Act. Accessed, August 29, 2002. Available from: http://www.cms.gov.schip/chontn 99.pdf.

12. Department of Health. Building a safe NHS. London: Department of Health; AC. $2000 ; 250-260$.

13. Council of the Institute of Medicine. American's Health in transition: protecting and improving quality. Washington. DC: National Academy Press; 1994. $213-220$.

14. John B. Working with community based groups: A model from the Philippine Health Insurance Corporation. International Conference on Health Insurance in Developing Countries, Berlin, (2005): $512-520$.

15. Campbell $P$, Lawthers AG. Improving the quality of health services: 
International health systems program, Harvard School of Public Health. Boston: (2005). Available from: http://www.harvard.edu/itsp/train.thml.

16. El-Zanaty F, Hussein E., Shawky G; Way A, Kisher S. Egypt Demographic and Health Survey (EDHS), (1995), Calverton Mary Land (USH): National Population Council (Egypt) and Macro international Inc. 2000. $210-215$.

17. Sakr ME Heiba AK. Health sector in Egypt: A Room for Improvement, Annual Meeting of the Human Development Capabilities Association, Netherland: 2006. $189-195$.

18. Harriagam JF. Over view of health services Scarborough ME: National Association of School Nurses. 2002; $114-23$.

19. Abd El-Fattah $\mathrm{H}$. The Health Insurance Organization of Egypt. An analytical review and strategy for reform, partnership for health reform, Technical Report No. 43.

20. Doughert D, Simpson L. Measuring the quality of children's health care: A Prerequisite to Action. 2004; 113 (1): $185-98$.

21. Mainz J. Defining and classifying clinical indicators for quality improvement. International Society for Quality in Health Care. International Journal for Quality in Health, 2005; 15 : $523-30$.

22. Hassaneen HR. Health education program for pregnant women on post partum care after normal deliveries. D.Ns thesis Faculty of Nursing, Assuit University. 2000. $118-135$.

23. Andrson CL. School health services in school health practice. $7^{\text {th }}$ ed. Saint Louis, London: CV Mosby; 1998. 184 190.

24. El-Maadawy N. Taha MK, Arafa M. Health Insurance book for students at school. Egypt: 1993. 5-14.
25. Myeni $A B, M C G r a t h ~ E$. Perspective in school health. Swaziland. J school. Health. 2000; 60:351-3.

26. WHO report of the 2000 joint committee on health education terminology. J School Health Education. 2001;33:143-6.

27. Chung PJ, Schuster MA. Access and quality in child health services: voltage drops. Archives of Disease in Children. 2004; 5, 77-87.

28. Clyat AR. School health services. J School Nurses. 2000; 11(3): 202-10.

29. Catalano K, ORY. J Initiative Nursing Spectrum. 1997: 7-17.

30. Waller L. Upper respiratory illness, recurrent infection, and day care center attendance. American of Respiratory and Critical Care Medicine. 2001; issue 55: $156-61$.

31. Lauber B. The common cold in children. J Gen Intern Med. 2005; 31: 229-36.

32. Pelletier J, Glynn EA, Broolin RH. Why the quality of the health care must be improved? Santa Monica, calif: RAND; 2002. $210-215$.

33. Rit G, Ravinderk. Recurrent abdominal pain in preschool children. $\mathrm{J} \mathrm{K}$ Science. 2004; 6(No)1: 31 - 3.

34. Peronic PG, pia Centiri GL. Predis posing factors for asthma. $\mathrm{J}$ of Clinical and Experimental Allergy. 2003; 33(10): 1349-54.

35. American Academy of Pediatrics. Role of the school nurses in providing school health services. 2004; issue No. 31: $401-5$.

36. Kersting DW, Mielck A. Accidents in preschool children in Boblingen district accidents. Cases and high risk group. Gesund Heitswesen. 2002; May, 63(5): $335-42$.

37. MOHP. Executive summary: Health sector reform program, Cairo: Technical Support Office; 1999. 115 120. 
38. Walker JR. Public policy and the supply of child care services. In: Blou M. (Ed.). The economics of child care New York: Russell Sage. 2001.75-85.

39. World Health Organization. Millennium development goals. (2006). Available from:http;//www.who.nt/mmd/goals/goa 14/cartext/en/index.html.

40. bethel $\mathrm{CH}$, Colleen $\mathrm{P}$, Edward $\mathrm{S}$. Assessing health system provision of well-child care. The promoting healthy developmental survey. $\mathrm{J}$ of Pediatrics. 2003; 107(5): 33-9.

41. Bassil A, Zaki, A, Zaher RS, El-sawy HI, Ahmed HM. Quality of care of children with chronic diseases in Alexandria, Egypt. The models of asthma, type I diabetes, epilepsy, and rheumatic heart disease. Pediatrics. 2000; 106(1): $12-32$.

42. Gunter K, Matteson D. Competency in pediatric nursing, A modular training program. London: Aspen publishers Co; 2001. 210-240.

43. Harriagan JF. Overview of school health services. Scarborough. ME. National Association of School Nurses. 2002; $412-18$.
44. United States Development of Health and Human Services. Healthy people 2010. understanding and improving health objectives for improving health. $2^{\text {nd }}$ ed. 2 volumes. Washington DC: US Government Printing Office; 2000. 91 102.

45. Tolljohann J. Effects of an in-services workshop on the health teaching self- efficiency of elementary school teaching. J of School Health. 1999; 60 (7): 261-5.

46. Al-Nasir AF, Sherman HJ. School teacher's knowledge of commen health problems in Bahrain. Eastern Mediterranean Health Journal. 2004; 10(5/5): 18-27.

47. Mark R. Chassin R, Robert W. Galvin $\mathrm{K}$. The national round table on the health care quality: the urgent need to improve health care on the health care quality. AMA. 2002; (280):1000-5.

48. Rajmil L, Barbara S, Carrel D. The quality care coverage in Catalonia, Spain. Arch Dis of Child. 2000; 83; 211-4. 